\title{
Azacitidine in patients with WHO-defined AML - Results of 155 patients from the Austrian Azacitidine Registry of the AGMT-Study Group
}

Lisa Pleyer ${ }^{1}$, Reinhard Stauder ${ }^{2}$, Sonja Burgstaller ${ }^{3}$, Martin Schreder $^{4}$, Christoph Tinchon $^{5}$, Michael Pfeilstocker ${ }^{6}$, Susanne Steinkirchner ${ }^{1}$, Thomas Melchardt ${ }^{1}$, Martina Mitrovic ${ }^{2}$, Michael Girschikofsky ${ }^{7}$, Alois Lang ${ }^{8}$, Peter Krippl ${ }^{9}$, Thamer Sliwa $^{10}$, Alexander Egle ${ }^{1}$, Werner Linkesch ${ }^{11}$, Daniela Voskova ${ }^{12}$, Hubert Angermann ${ }^{13}$ and Richard Greil ${ }^{1 *}$

\begin{abstract}
Objective: The Austrian Azacitidine Registry is a multi-center database (ClinicalTrials.gov: NCT01595295). The nature and intent of the registry was to gain a comprehensive view of the use, safety and efficacy of the drug in a broad range of AML-patients treated in real-life scenarios.

Patients and methods: The sole inclusion criteria were the diagnosis of WHO-AML and treatment with at least one dose of azacitidine. No formal exclusion criteria existed. A total of $155 \mathrm{AML}$-patients who were mostly unfit/ ineligible for intensive chemotherapy, or had progressed despite conventional treatment, were included. True ITT-analyses and exploratory analyses regarding the potential prognostic value of baseline-variables/performance-/ comorbidity-/risk-scores on overall survival (OS), were performed.

Results: In this cohort of 155 pretreated (60\%), and/or comorbid (87\%), elderly (45\% $\geq 75$ years) AML-patients, azacitidine was well tolerated and efficacious, with an overall response rate $(C R, m C R, P R, H I)$ of $45 \%$ in the total cohort (ITT) and 65\% in patients evaluable according to IWG-criteria, respectively. Pre-treatment with conventional chemotherapy $(P=.113)$, age $\leq />80$ years $(P=.853)$, number of comorbidities $(P=.476)$, and bone marrow (BM) blast count $(P=.663)$ did not influence OS. In multivariate analysis hematologic improvement alone (without the requirement of concomitant bone marrow blast reduction), although currently not regarded as a standard form of response assessment in AML, was sufficient to confer OS benefit (18.9 vs. 6.0 months; $P=.0015)$. Further deepening of response after first response was associated with improved OS (24.7 vs. 13.7 months; $P<.001$ ).
\end{abstract}

Conclusions: In this large cohort of AML-patients treated with azacitidine, age $>80$ years, number of comorbidities and/or BM-blasts $>30 \%$ did not adversely impact OS.

Keywords: Austrian azacitidine registry, Azacitidine, AML, Overall survival, Prognostic factors, Bone marrow blasts

\section{Background}

Acute myeloid leukemia (AML) is an aggressive disease with an unfavorable prognosis $[1,2]$. Only approximately $1 / 3$ of elderly AML-patients are eligible for intensive chemotherapeutic approaches. Results remain poor and unsatisfactory, even for those who do meet inclusion

\footnotetext{
* Correspondence: r.greil@salk.at

'3rd Medical Department with Hematology and Medical Oncology, Hemostaseology, Rheumatology and Infectious Diseases, Laboratory for Immunological and Molecular Cancer Research, Oncologic Center, Paracelsus Medical University Hospital Salzburg, Müllnerhauptstrasse 48, Salzburg 5020, Austria

Full list of author information is available at the end of the article
}

criteria for such treatment (induction mortality of up to 29\%; 2 year OS 6-11\%) [3-5]. Patients not suitable for intensive chemotherapy due to high age, comorbidities, poor performance status and/or adverse cytogenetics, or who have failed these treatment options, are frequently offered best supportive care (BSC) only [6], and the prognosis is dismal [7]. Other treatment options such as low-dose cytarabine, topotecan, gemtuzumab ozogamicin, clofarabine, cloretazine, or tipifarnib have not been promising [8-14]. Treatment options for AML-patients with $>30 \%$ BM-blasts unfit/ineligible for intensive chemotherapeutic approaches are particularly limited, as hypomethylating

\section{Biomed Central}


agents were not approved for this patient group. Only very recently has decitabine been granted EMA-, but not FDAapproval for the treatment of WHO-AML patients aged 65 or older who are not candidates for standard induction chemotherapy (http://www.ema.europa.eu/ema/index.jsp? curl=pages/medicines/human/medicines/002221/human_ med_001589.jsp\&mid=WC0b01ac058001d124).

Azacitidine $\left(V^{2}\right.$ idaza $^{\circ}$ ) received FDA-approval for MDS and AML with 20-30\% BM-blasts in 2004 based on the results of several CALGB-trials [15]. EMA-approval for HR-MDS and AML with 20-30\% BM-blasts was granted in 2008 following the pivotal AZA-001 trial [16]. In a subset-analysis, azacitidine was shown to prolong OS in elderly AML-patients with 20-30\% BM-blasts [17].

The Austrian Azacitidine Registry (AAR) (ClinicalTrials. gov: NCT01595295) comprises 155 AML-patients who did not qualify for intensive chemotherapeutic approaches in most cases, and is currently the largest cohort of AMLpatients treated with azacitidine published to date, with the highest per capita coverage of AML-patients in a nationwide registry, suggesting limited selection (Additional file 1: Tables S1 and S2). The aim was to include most AML-patients treated with azacitidine in Austria, and to gain a comprehensive view of the use, safety and efficacy of the drug in a broad range of WHO-defined AML, including patients with $>30 \%$ bone marrow blasts, who have limited other therapeutic options, and for whom the drug has not been approved yet.

\section{Results}

\section{Patient characteristics}

Between 02/2009 and 02/2012, 155 AML-patients from 12 specialized centers for hematology and medical oncology were included; No patients were excluded from the analyses (Additional file 2: Figure S1A). Patient baseline characteristics can be taken from Table 1. Median age was 73 (range 33-91); 57\% of patients were older than $75,23 \%$ were older than 80 and $8 \%$ were older than 85 years, respectively; 98 patients $(63 \%)$ had $>30 \%$ BMblasts; $17 \%$ had an unfavorable karyotype, and $74 \%$ had an intermediate karyotype according to MRC-criteria [18]. In the absence of consensus for cytogenetic classification of AML in the elderly, we additionally assessed the IPSS cytogenetic risk categories (Table 1).

\section{Treatment modalities}

Azacitidine was administered as first line therapy in 41\% of patients. Bridging to allogeneic stem cell transplantation (allo-SCT), maintenance after complete response (CR) to chemotherapy, no CR to, or early relapse after allo-SCT or conventional chemotherapy was the reason for treatment in $3 \%, 4 \%, 3 \%$ and $29 \%$ of patients, respectively (Table 1). Azacitidine was not always second line therapy in chemotherapy refractory patients, but
Table 1 Baseline characteristics

\begin{tabular}{lc}
\hline Median age, years (range) & $73(33-91)$ \\
WHO diagnosis*, $\mathbf{n}(\%)$ & $16(10.3)$ \\
t-AML & $16(10.3)$ \\
AML-RCA & $88(56.8)$ \\
AML-MRF only & $13(8.4)$ \\
AML-MRC & $58(37.4)$ \\
Preexisting MDS/MPN or MLD & $17(11.0)$ \\
Preexisting MDS/MPN or MLD + MRC & $35(22.6)$ \\
AML-NOS
\end{tabular}

Peripheral blood blasts, $\mathrm{n}(\%)$

$0 \%$

$58(37.4)$

$>0 \%$

Mean,\%

$97(62.6)$

14

Median (range),\%

$3(0-90)$

Bone marrow blasts, $\mathrm{n}(\%)$

$<20 \% \dagger$

$26(16.8)$

$20-30 \%$

$31(20.0)$

$>30 \%$ (off label use)

98 (63.2)

Mean,\%

42

Median (range), $\%$

35 (0-98)

White blood cell count, $\mathbf{n}(\%)$

Non-MP-AML $(<10 \mathrm{G} / \mathrm{l})$

$122(78.7)$

MP-AML (> $10 \mathrm{G} / \mathrm{l})$

$33(21.3)$

Transfusion dependence (TD), $\mathbf{n}(\%)$

Any type of TD

$101(65.2)$

RBC-TD

$97(62.6)$

PLT-TD

$60(38.7)$

RBC-TD + PLT-TD

$56(36.1)$

IPSS cytogenetic risk $\neq$, n (\%)

Not evaluable

$11(7.1)$

Good

$91(58.7)$

Intermediate

$26(16.1)$

Poor

$27(17.4)$

MRC cytogenetic risk $\neq, \mathrm{n}(\%)$

Not evaluable

$11(7.1)$

Good

$3(1.9)$

Intermediate

$115(74.2)$

High

$26(16.8)$

Comorbidities, n (\%)

Thromboembolic episodes 21 (13.5)

Renal insufficiency 29 (18.7)

Liver disease $\quad 20$ (12.9)

Diabetes mellitus $26(16.8)$

Coronary artery disease $\quad 34(21.9)$ 
Table 1 Baseline characteristics (Continued)

\begin{tabular}{|c|c|}
\hline COPD & $15(9.7)$ \\
\hline Prior/concomitant malignancies & $35(22.6)$ \\
\hline \multicolumn{2}{|l|}{ Number of comorbidities, $\mathrm{n}(\%)$} \\
\hline $0-1$ & $66(42.6)$ \\
\hline $2-3$ & $61(39.4)$ \\
\hline$>3$ & $28(18.1)$ \\
\hline \multicolumn{2}{|l|}{ ECOG Prognostic Score, n (\%) } \\
\hline ECOG $<2$ & $114(73.6)$ \\
\hline$E C O G \geq 2$ & $41(26.4)$ \\
\hline \multicolumn{2}{|l|}{ HCT-Cl, n (\%) } \\
\hline Low risk & $46(29.7)$ \\
\hline Int. risk & $46(29.7)$ \\
\hline High risk & $50(32.2)$ \\
\hline No data & $13(8.4)$ \\
\hline \multicolumn{2}{|l|}{ Treatment prior to azacitidine $\S, \mathbf{n}(\%)$} \\
\hline None & $63(40.6)$ \\
\hline Erythropoietin stimulating agents & $15(9.7)$ \\
\hline G-CSF & $19(12.3)$ \\
\hline Thrombopoietin stimulating agents & $1(0.7)$ \\
\hline Iron chelation therapy & $5(3.2)$ \\
\hline Thalidomide & $3(1.9)$ \\
\hline Lenalidomide & $6(3.9)$ \\
\hline Low-dose cytarabine & $10(6.5)$ \\
\hline Intensive chemotherapy & $60(38.7)$ \\
\hline Others & $16(10.3)$ \\
\hline \multicolumn{2}{|l|}{ Reason for treatment, $\mathrm{n}(\%)$} \\
\hline 1st line treatment & $63(40.6)$ \\
\hline Bridging to allogeneic $\mathrm{SCT}{ }^{\#}$ & $4(2.6)$ \\
\hline Maintenance after CR to conventional chemotherapy & $6(3.9)$ \\
\hline No CR to/early relapse after conventional chemotherapy & $45(29.0)$ \\
\hline No CR to/early relapse after allogeneic SCT & $5(3.2)$ \\
\hline No CR to other prior treatment & $32(20.6)$ \\
\hline
\end{tabular}

t-AML indicates treatment related AML; AML-RCA, AML with recurrent cytogenetic abnormalities; AML-MRF, AML with MDS related features; MPN, myeloproliferative neoplasia; MLD, multilineage dysplasia; MRC, MDS-related cytogenetics; AML-NOS, AML not otherwise specified; MP, myeloproliferative; COPD, chronic obstructive pulmonary disease; NHL, non-Hodgkin's lymphoma; ECOG, Eastern Cooperative Oncology Group; CR, complete response; SCT, stem cell transplantation;

* If a patient fulfilled criteria for more than one WHO-category, weighting was performed as follows: $t-A M L>A M L-R C A>A M L-M R F$.

tBM-blast count was $<20 \%$, in those patients with established AML who were refractory to, -or had no $\mathrm{CR}$ after-, conventional chemotherapy or allogeneic stem cell transplantation.

¥Pre-treatment cytogenetics were available in $92 \%$ of patients and were determined by conventional metaphase karyotyping (46\%), interphase-FISH $(16 \%)$, or both (38\%).

§Numbers may add up to $>100 \%$ as multiple selections were possible. "None of the patients for whom azacitidine was intended as bridging was in complete remission to prior therapies, and all patients had relapsed after multiple lines of intensive chemotherapy; only one of these patients proceeded to allogeneic SCT (the others died whilst still on, or within 8 weeks after termination of azacitidine treatment due to disease progression and/or infectious complications). also third, fourth, fifth or last line therapy for a relevant proportion of our patients (28\%).

Azacitidine dose, application route, and administration schedule were exclusively based on the risk/benefit-estimation of the treating physician. The median and mean number, as well as range of azacitidine courses was 4, 6.3 and 1-24, respectively. Most cycles (90\%) were applied subcutaneously (average dose/cycle $814 \mathrm{mg}$ ); 10\% were given intravenously (average dose/cycle $870 \mathrm{mg}$ ); $79 \%$ of patients predominantly received 7 days of azacitidine (57\% FDA-approved d1-7 (median dose/ cycle $924 \mathrm{mg}$ ), 22\% 5-2-2 (median dose/cycle $900 \mathrm{mg}$ )) (Additional file 1: Table S3). FDA-approved azacitidine target-dose $\left(75 \mathrm{mg} / \mathrm{m}^{2} \times 7+/-10 \%\right)$ was reached in $53 \%$ of applied cycles; 214/958 (22\%) of all cycles were administered as 'flat' dosage (i.e. $100 \mathrm{mg}$ azacitidine/cycleday; median dose/cycle $700 \mathrm{mg}$ ). Dose reduction of azacitidine due to an adverse event (AE) was necessary in $28(18 \%)$ patients.

\section{Concomitant treatment and best supportive care measures}

In all 958 azacitidine cycles applied to AML-patients, erythropoietin stimulating agents (ESA) (3\%), iron chelation treatment (ICT) (3\%), and G-CSF (21\%) were given in parallel to azacitidine when deemed necessary by the treating physician. ESA and ICT were rarely required after cycle 5, whereas G-CSF-usage did not show the same decline.

\section{Response}

Overall response, defined as complete response (CR), marrow response (mCR), partial response (PR) and hematologic improvement (HI), was documented in $45.2 \%$ of the total intention-to-treat (ITT) cohort and in $65.4 \%$ of patients evaluable according to IWG-criteria [19] (i.e. had received $>2$ cycles of azacitidine); Hematologic improvement was documented in 32\% (ITT) and $46 \%$ (IWG), respectively; Best marrow response [20] is shown in Table 2.

Taking a closer look at responding patients $(n=70)$, $77 \%$ received 7 days of azacitidine in the first cycle $(65 \%$ $\mathrm{d} 1-7,13 \% 5-2-2)$ and $82 \%$ predominantly received 7 days of azacitidine over all cycles (52\% d1-7 (median dose/ cycle $871 \mathrm{mg}$ ), 30\% 5-2-2 (median dose/cycle $837 \mathrm{mg}$ ) (Additional file 1: Table S3). Of note, the distribution of applied schedules, as well as the median and mean azacitidine dosages/cycle did not differ between responders and non-responders (Additional file 1: Table S3). The median and mean number, as well as range of cycles received by responding patients is $9,10.3$ and 1-31, respectively. 
Table 2 Response to azacitidine $[18,19]$

\begin{tabular}{|c|c|}
\hline \multicolumn{2}{|c|}{ Transfusion independence, $n,(\%$ ITT*), [\% IWG +$]$} \\
\hline PLT-TI & $24 / 60(40.0), 24 / 43[55.8$ \\
\hline $\mathrm{RBC}-\mathrm{TI}$ & $35 / 97(36.1), 35 / 69[50$. \\
\hline \multicolumn{2}{|c|}{ Hematologic improvement, n, (\% ITT), [\% IWG $\neq]$} \\
\hline $\mathrm{No} \mathrm{HI}$ & $58(37.4)[54.2]$ \\
\hline HI-Any & 49 (31.6) [45.8] \\
\hline \multicolumn{2}{|l|}{ Best marrow response, $\mathrm{n}(\% \mathrm{ITT})[\%$ eval.§] } \\
\hline$C R$ & $15(9.7)[20.0]$ \\
\hline $\mathrm{mCR}$ & $5(3.2)[6.7]$ \\
\hline PR & $32(20.6)[42.7]$ \\
\hline mSD & $19(12.3)[25.3]$ \\
\hline Primary PD & $4(2.6)[5.3]$ \\
\hline Overall response, $n,(\%$ ITT), [\% IWG $\neq$ ] & $70(45.2)[65.4]$ \\
\hline$C R$ & $15(9.7)[14.0]$ \\
\hline $\mathrm{mCR}$ & $5(3.2)[4.7]$ \\
\hline PR & $32(20.6)$ [29.9] \\
\hline mSD with $\mathrm{HI}$ & $4(2.6)[3.7]$ \\
\hline HI only & $14(9.0)[13.1]$ \\
\hline No response & 61 (39.4), 37 [34.6] \\
\hline
\end{tabular}

IWG indicates International Working Group Criteria; TI, transfusion independence; HI, hematologic improvement; $\mathrm{mCR}$, marrow CR; mSD, marrow stable disease; $\mathrm{BMP}$, bone marrow puncture; PD, progressive disease;

${ }^{*}$ Concerns all patients that were transfusion dependent at baseline;

tConcerns number of patients that were transfusion dependent at baseline and received $>2$ cycles of azacitidine and were thus evaluable for response assessment according to IWG-criteria;

\#Evaluable according to IWG-criteria, i.e. patients that received $>2$ cycles of azacitidine $(n=107)$;

$\S$ Concerns patients in whom bone marrow puncture was performed $(n=75)$; bone marrow assessment was performed as clinically necessary, and at the discretion of the respective treating physician; a total of 140 bone marrow punctures were performed in 75 patients (of patients in whom no bone marrow puncture was performed, 30 received only one and 18 received only two cycles of azacitidine; of these, 26 died within 2 months and a further 11 died within 6 months after stop of azacitidine treatment).

\section{Time to response and response deepening}

Median (mean) time to first response was 4 (3.5) months. First response occurred after 3, 4 and 5 cycles in $44 \%, 77 \%$ and $87 \%$ of responding patients, respectively, but could be observed as late as cycle 10. First response was best response in $46 / 70$ patients (66\%). Further deepening of response after first response was seen in 24/70 (34\%) of responders. Best response was reached by cycle 8 in 93\%, but could be observed as late as cycle 19. Median (mean) time from first to best response was 2.8 (3.6) months, respectively.

\section{Toxicity and adverse events}

A total of 501 adverse events were documented in 958 azacitidine cycles. The number of adverse events declined continuously from $\sim 40 \%$ of patients in the $1^{\text {st }}$ cycle to $<20 \%$ as of cycle $\geq 10$. Overall, $32 \%$ of all adverse events and $20 \%$ of grade $3-4$ adverse events were attributed to azacitidine; $22 \%$ resulted in hospitalization, $6 \%$ resulted in death; $69 \%$ had no consequence for azacitidine treatment; $6 \%, 14 \%, 9 \%$, and $1 \%$ resulted in dose reduction, treatment pause, termination of treatment, or prolongation of azacitidine cycle duration $>28$ days, respectively.

Grade 3-4 neutropenia, thrombocytopenia, and anemia were documented in $32 \%, 25 \%$, and $21 \%$ of patients, respectively; clinically relevant bleeding events were noted in 9\% (Table 3). Non-hematologic toxicity was usually mild, the most common adverse events being fatigue (42\%), unspecified pain $(30 \%)$, gastro-intestinal (26\%), and injection site reactions (21\%). Infectious complications of any grade were documented in 63\%, febrile neutropenia in $18 \%$ (Table 3). Grade 3-4 events occurred in 29\% and were dominated by pulmonary infections, sepsis, and CMVreactivations. Hospital admission was required in $43 \%$ and transfer to an intensive care unit was necessary in $4 \%$.

Only 18 non-hematologic grade 3-4 events occurred in 16 patients (10\%): $15 / 18$ of these grade $3-4$ events occurred in the cardiac system: left-ventricular output failure $(n=10)$, arrhythmia $(n=4)$, cardiac ischemia $(n=1)$. In 10/15 (67\%) patients experiencing cardiac grade 3-4 events, pre-existing coronary artery disease, reduced 
Table 3 Specific adverse events*, $\uparrow$

\begin{tabular}{|c|c|c|c|}
\hline Variable & Grade & n pts., (\%) & n total events \\
\hline Hematologic toxicity ${ }^{\#}$ & G3-4 & $69(44.5)$ & 148 \\
\hline Thrombopenia & G3-4 & $38(24.5)$ & 87 \\
\hline Neutropenia & G3-4 & 49 (31.6) & 99 \\
\hline Anemia & G3-4 & $33(21.3)$ & 80 \\
\hline Bleeding events & - & $14(9.0)$ & 31 \\
\hline Febrile neutropenia & - & $28(18.1)$ & 46 \\
\hline \multirow[t]{5}{*}{ Infectious complications } & Total & $98(63.2)$ & 256 \\
\hline & G1 & $14(9.0)$ & 85 \\
\hline & G2 & $39(25.2)$ & 107 \\
\hline & G3 & $16(10.3)$ & 26 \\
\hline & G4 & $29(18.7)$ & 38 \\
\hline \multicolumn{4}{|l|}{ Non-hematologic toxicity } \\
\hline \multirow[t]{2}{*}{ Liver } & G1-2 & $1(0.6)$ & 1 \\
\hline & G3-4 & $0(0.0)$ & 0 \\
\hline \multirow[t]{2}{*}{ Kidney } & G1-2 & $5(3.2)$ & 5 \\
\hline & G3-4 & $0(0.0)$ & 0 \\
\hline \multirow[t]{2}{*}{ Heart } & G1-2 & $3(1.9)$ & 5 \\
\hline & G3-4 & $13(8.4)$ & 15 \\
\hline \multirow[t]{2}{*}{ Blood pressure } & G1-2 & $2(1.3)$ & 2 \\
\hline & G3-4 & $1(0.6)$ & 1 \\
\hline \multirow[t]{2}{*}{ Metabolic } & G1-2 & $1(0.6)$ & 1 \\
\hline & G3-4 & $0(0.0)$ & 0 \\
\hline \multirow[t]{2}{*}{ Thromboembolic } & G1-2 & $9(5.8)$ & 10 \\
\hline & G3-4 & $1(0.6)$ & 1 \\
\hline \multirow[t]{2}{*}{ Neurologic } & G1-2 & $11(7.1)$ & 18 \\
\hline & G3-4 & $1(0.6)$ & 1 \\
\hline \multirow[t]{2}{*}{ Nausea } & G1-2 & $16(10.3)$ & 24 \\
\hline & G3-4 & $0(0.0)$ & 0 \\
\hline \multirow[t]{2}{*}{ Vomiting } & G1-2 & $4(2.6)$ & 5 \\
\hline & G3-4 & $0(0.0)$ & 0 \\
\hline \multirow[t]{2}{*}{ Constipation } & G1-2 & $3(1.9)$ & 5 \\
\hline & G3-4 & $0(0.0)$ & 0 \\
\hline \multirow[t]{2}{*}{ Diarrhea } & G1-2 & $17(11.0)$ & 25 \\
\hline & G3-4 & $0(0.0)$ & 0 \\
\hline \multirow[t]{2}{*}{ GIT-others } & G1-2 & $11(7.1)$ & 11 \\
\hline & G3-4 & $0(0.0)$ & 0 \\
\hline \multirow[t]{2}{*}{ Injection site reaction } & G1-2 & $32(20.6)$ & 48 \\
\hline & G3-4 & $0(0.0)$ & 0 \\
\hline \multirow[t]{4}{*}{ Fatigue } & Total & $65(41.9)$ & 99 \\
\hline & Relieved by rest & $24(15.5)$ & 50 \\
\hline & Not relieved by rest & $25(16.1)$ & 32 \\
\hline & Limiting self care & $16(10.3)$ & 17 \\
\hline \multirow[t]{2}{*}{ Pain } & Total & $46(29.7)$ & 78 \\
\hline & Mild & $25(16.1)$ & 52 \\
\hline
\end{tabular}


Table 3 Specific adverse events*, + (Continued)

\begin{tabular}{|c|c|c|c|}
\hline & Moderate & 18 (11.6) & 23 \\
\hline & Severe & $3(1.9)$ & 3 \\
\hline \multirow[t]{3}{*}{ Surgery } & Total & $20(12.9)$ & 24 \\
\hline & Elective & $13(8.4)$ & 16 \\
\hline & Emergency & $7(4.5)$ & 8 \\
\hline \multirow[t]{3}{*}{ Fall } & Total & $14(9.0)$ & 16 \\
\hline & With fracture & $8(5.2)$ & 9 \\
\hline & With hemorrhage & $5(3.2)$ & 8 \\
\hline Novel solid tumor & Yes & $3(1.9)$ & 3 \\
\hline
\end{tabular}

cardiac function or arrhythmias were documented prior to azacitidine treatment, and worsening was not thought to be azacitidine-related.

\section{Overall survival and potential prognostic parameters}

Median OS was 16.3 (95\% CI 12.23-19.97) months as of first diagnosis, and 9.8 (95\% CI 8.59-10.93) months as of treatment start with azacitidine. Median (mean) time from first diagnosis to treatment start with azacitidine for untreated $(n=63)$ versus pretreated $(n=92)$ patients was 0.6 (4.1) and 8.5 (14.9) months, respectively. Median (mean) time from initial diagnosis to azacitidine treatment for patients who received no, one, or multiple lines of conventional chemotherapy prior to azacitidine, was 0.7 (6.7), 1.8 (4.1), and 13.3 (21.7) months, respectively. 48 patients received $\leq 2$ cycles of azacitidine; 23 of these died within 1 month of treatment termination, and a further 14 died within 6 months. Median (mean) time from azacitidine treatment stop to death was 1.8 (4.0) months, respectively.

In multivariate analysis the following baseline parameters had a significant effect on overall survival: peripheral blood blasts $(P=0.0398)$ and ECOG performance score (ECOGPS) $(P=0.0397)$ (Figure 1 and Additional file 1: Table S5). In univariate analysis patients with adverse cytogenetics $(-7 q,-7$, abn (3q), complex karyotype) $(n=28)$ had worse OS than patients with other cytogenetic abnormalities or normal karyotype $(\mathrm{n}=115)$ (5.1 vs. 10.5 months, $P=0.009)$ (Additional file 1: Table S5). Patients pre-treated with 'imids' ( $n=9)$, i.e. thalidomide or lenalidomide, may have worse OS: prior treatment with either of these substances was associated with a significant adverse effect on survival ( $\mathrm{P}=0.008$, median OS 3.0 vs. 9.7 months, respectively) (Additional file 1: Table S5). However, the number of patients pretreated with thalidomide/lenalidomide was too small to establish strong conclusions or to perform

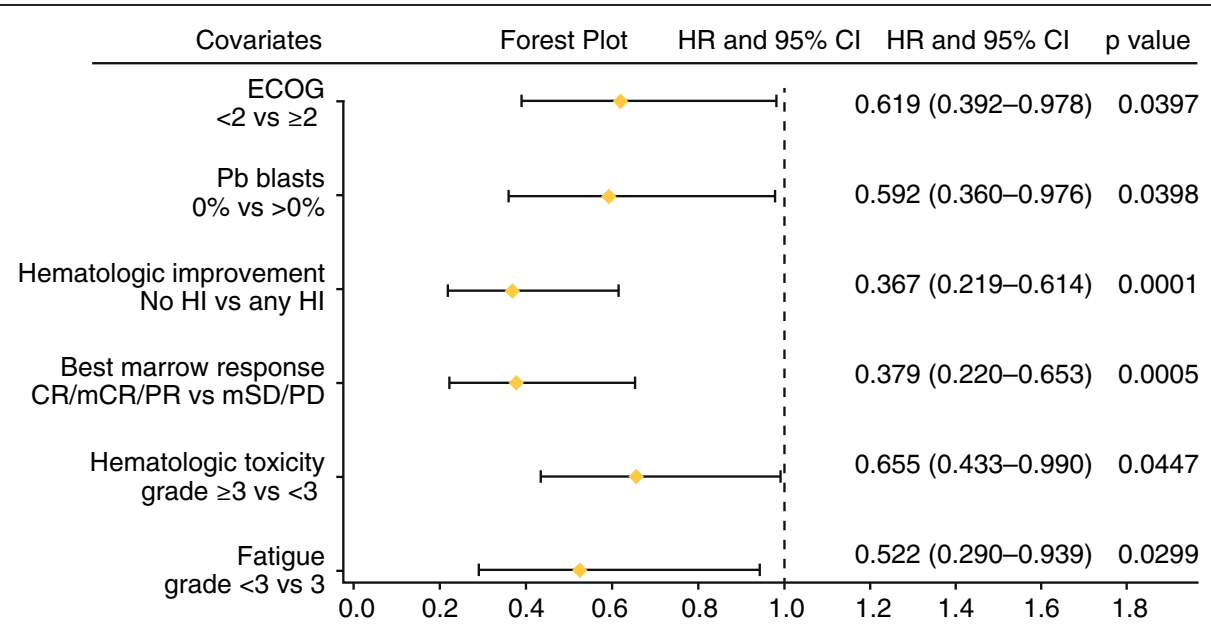

Figure 1 Factors significantly influencing overall survival in multivariate analysis. 
multivariate analysis. Of the 6 patients pretreated with lenalidomide, 2 had preceeding MDS with $-5 q$ as sole aberration, one had a complex karyotype including $-5 \mathrm{q}$, one had a complex karyotype including monosomy 5, one had a normal karyotype, and in one patient karyotype was not evaluable.

The absolute number of comorbidities did not adversely affect OS of AML-patients treated with azacitidine with a cut-off of $<3 / \geq 3$ comorbidities $(P=0.151)$. When a cut-off of $<4 / \geq 4$ comorbidities was analyzed, there was trend for reduced OS, which was however not statistically significant $(P=0.086)$ (Additional file 1: Table S4). MRCcytogenetic risk groups could not predict overall survival, however a trend was noted $(P=0.093)$.

Baseline factors that did not significantly affect OS in univariate analyses (Additional file 1: Table S4) include WBC $\leq />10 G / 1(P=0.346), \mathrm{LDH} \leq />225 \mathrm{IU} / \mathrm{l}(P=0.123), \mathrm{S}-\mathrm{EPO}$ level $(P=0.661), \quad$ RBC-TD $(P=0.542), \quad$ and PLT-TD $(P=0.149)$. Age had no adverse effect on outcome of AML-patients treated with azacitidine, irrespective of whether a cut-off of $</ \geq 75(P=0.174)$ or $</ \geq 80$ years was chosen $(P=0.853)$ (Figure 2a). Bone marrow blast count $\leq 30 />30 \%$ had no adverse effect on OS, irrespective of whether the whole cohort $(P=0.663)$ was analyzed, or whether patients with prior intensive CTX $(P=0.313)$, or all pretreated patients were excluded $(P=0.127)$ (Figure $2 \mathrm{~b}$, Additional file 1: Table S4). Prior treatment with ESA ( $P=$ 0.873), G-CSF $(P=0.841)$, low-dose cytarabine $(P=0.630)$ or one or several lines of conventional chemotherapy $(P=$ 0.113 ) had no adverse effect on OS (Additional file 1: Table S4). In concordance, patients, for whom absence of CR or refractoriness to prior intensive chemotherapy was the reason for treatment with azacitidine, did not have worse OS $(P=0.268)$.

In multivariate analysis the following response related factors had a significant effect on overall survival: hematologic improvement $(P=0.0001)$ and marrow response $(P=0.0005)$ (Figure 1 and Additional file 1: Table S5). Any kind of response resulted in significantly longer OS, be it marrow response ( 24.7 vs. 15.2 vs. 2.3 months

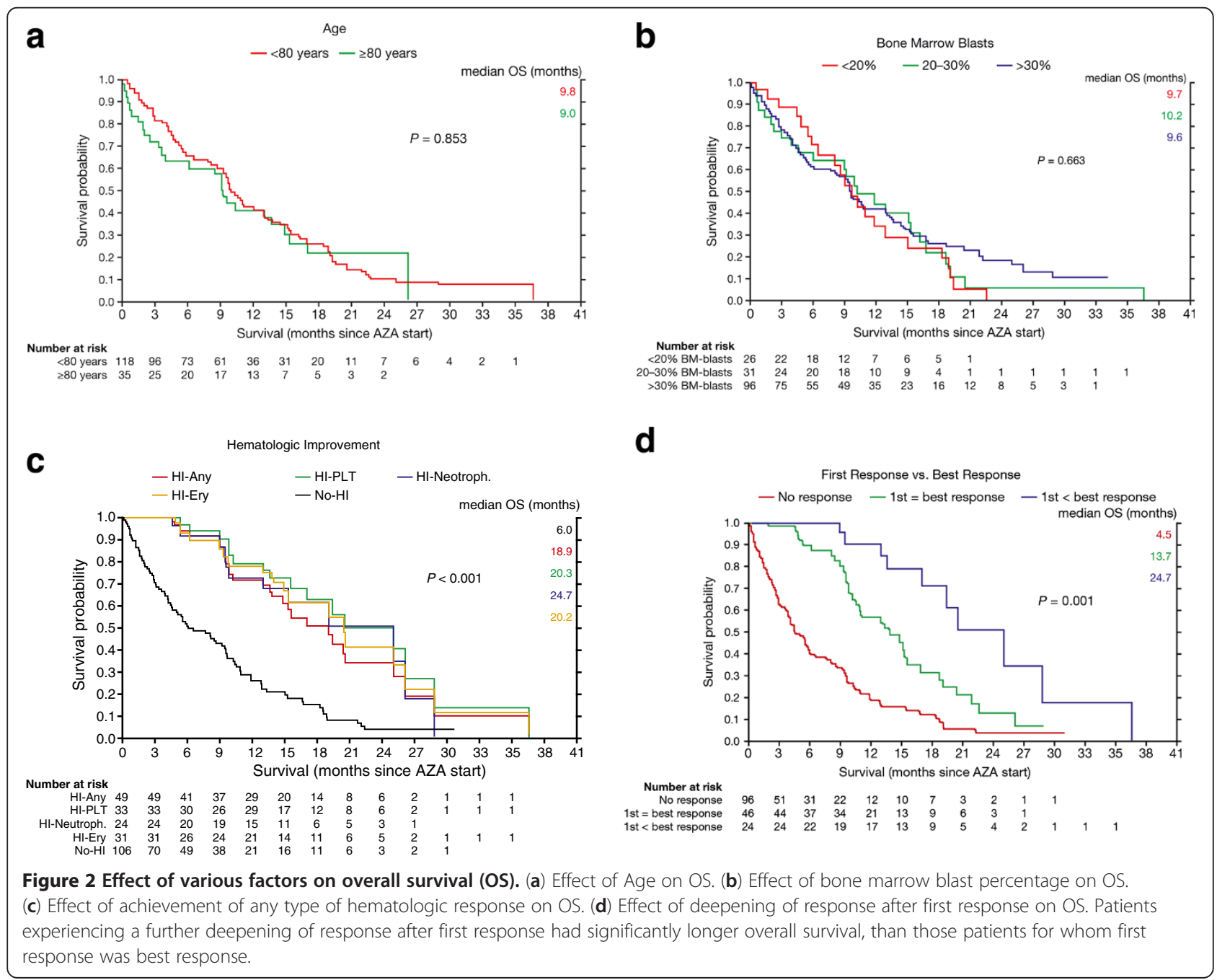


for CR vs. mSD vs. PD), achievement of PLT-TI (13.7 vs. 8.9 months), RBC-TI (19.3 vs. 9.6 months), or $\mathrm{HI}$ in one or all cell lineages (18.9 vs. 6.0 months) (Figures 1 and 2c, Additional file 1: Table S5). Continued azacitidine beyond first response resulted in further deepening of response in $34.3 \%$ of responders, which translated into significantly longer OS (24.7 months), compared with patients for whom first response was best response (13.7 months) $(P<0.001)$ (Figure 2d, Additional file 1: Table S5).

In multivariate analysis the occurrence of fatigue $(P=0.0299)$ or hematologic toxicity $(P=0.0447)$ as adverse events, had an independent prognostic effect on OS (Figure 1 and Additional file 1: Table S5). Azacitidine dose or schedule and the occurrence and duration of various non-hematologic adverse events had no negative impact on OS (Additional file 1: Table S4).

\section{Discussion}

With 155 AML-patients included in the AAR and 8.4 million inhabitants, this is the highest per capita coverage of AML-patients in a nationwide registry, suggesting limited selection. The per capita inclusion of AML-patients into our national registry (18.9 per million inhabitants) is the largest as compared to data from other countries that has so far been published (0.06-6.58 per million inhabitants) (Additional file 1: Table S1) [15,17,21-26]. The number of AML diagnoses per year, as well as the number of AML-patients included in the respective year for Austria in general and Salzburg in particular, is presented in Additional file 1: Table S2 (data obtained from Statistics Austria, personal communication 14.03.13). The percentage of patients in the MRC cytogenetic high-risk category is $17 \%$ in our AML cohort, which seems less than the proportion of cytogenetic high-risk AML-patients reported in several, but not all previously published smaller AMLcohorts treated with azacitidine (Additional file 1: Table S1). Cumulating evidence suggests that patients with high-risk cytogenetics respond poorly to conventional chemotherapeutic approaches, and that these patients especially may be candidates for hypomethylating treatment. Therefore, according to our understanding, patients with high-risk cytogenetics should be overrepresented, -rather than underrepresented-, in underrepresented-, in our registry, if an explicit selection bias concerning cytogenetic risk categories were present.

Median age, age range, pretreatment cytogenetic risk groups, azacitidine schedule and median azacitidine cycles are comparable to those documented in much smaller AML-patient-subsets $(n=20-82)$ in almost all previously published clinical trials and observational data so far (Additional file 1: Table S1) Therefore we believe that selection bias is minimized, both with respect to the choice of AML-patients for azacitidine treatment, as well as for the decision to include the patients in the registry (Additional file 2: Figure S2A).

The most common hematologic and non-hematologic adverse events, and the rates thereof are almost identical to previously published data from controlled trials as well as retrospective analyses $[16,27]$. CR/mCR-, as well as HIrates are comparable to, -and PR-rates slightly higher than-, the rates obtained in smaller series $(n=20-82)$ of AML-patients treated with azacitidine by others [15,17,21-25]. Due to higher PR-rates, the overall response rate (Table 2) is one of the highest documented for AMLpatients treated with azacitidine so far.

Median OS of elderly AML-patients is still low, irrespective of treatment modality. Conventional chemotherapy and other treatment options such as low-dose cytarabine, topotecan, gemtuzumab ozogamicin, clofarabine, cloretazine, tipifarnib, or laromustine show median OS rates of 2.0-6.7 months [7-13,28-31]. In comparison, AMLpatients with $20-30 \%$ blasts (formerly RAEB-t) treated with BSC only $(n=223$; median age $73 a)$ had a median OS of 5.2 months in a recent update of the Düsseldorf MDS registry data (U.Germing, personal communication, 17/09/2012). This is similar to 5.0 months OS of AML-patients randomized to best supportive care or low-dose cytarabine in a recent phase-III trial of untreated AML [32]. Therapy-naïve AML-patients $(n=242)$ randomized to decitabine in the same trial had a median OS of 7.7 months, which led to a positive opinion by EMA, with formal approval expected soon [32]. Previous reports of much smaller series of AMLpatients treated with azacitidine documented median OS rates of 3, 7-9, 2.5-15, 8, 6-16 and 8.8 months, respectively [21-25,30]. (The reports giving OS-ranges did not separate the OS of AML- and MDS-patients explicitly). In light of the above, our OS results of 9.8 months are encouraging. Only two subanalysis of randomized clinical trials (CALGB$9211(\mathrm{n}=27)$, AZA-001 $(\mathrm{n}=55)$ ) reported longer OS than observed in our cohort $[17,33]$. The authors of the latter stated that this is likely due to exclusion of patients with an estimated life expectancy $<3$ months, ECOG-PS $>2$, t-AML, prior treatment, or planned allogeneic SCT $[16,17]$. Overall, $61 \%$ of our registry population would have been excluded from this trial due to these reasons alone; if BM-blast count $>30 \%$ is also taken into account, $90 \%$ would have been excluded. We thus consider our OS data to be remarkable, particularly since they were observed in a real-life AMLpatient cohort, not selected or restricted by stringent inclusion/exclusion-criteria, and in which most patients had no other treatment option than azacitidine.

Significantly longer OS was observed in patients achieving any kind of response, be it marrow response, achievement of RBC/PLT-TI, or HI (Figures 1 and 2c, Additional file 1: Table S5). Similar results have been shown in smaller AML-patient cohorts $(\mathrm{n}=11)$ treated with azacitidine [34]. Others, who did not analyze the HI-rate 
in their AML-cohorts ( $\mathrm{n}=25-55$, BM-blasts 20-30\%), also noted, that azacitidine can provide survival benefit, despite a low CR-rate [17], a finding which is similar to that seen in high-risk MDS-patients [16]. However, it is for the first time shown in multivariate analysis, that response in terms of $\mathrm{HI}$ alone, without requirement of concomitant BM-blast reduction, seems sufficient to confer longer OS in AMLpatients treated with azacitidine (18.9 vs. 6.0 months) (Figure 1). If the commonly used AML response criteria [20] were to be applied, patients who experience HI without simultaneous BM-blast reduction would be considered as having neither CR nor PR, and thus as non-responders, with the result that treatment with azacitidine would/might be discontinued. In our opinion these patients should be considered as responders, and should continue to receive azacitidine until unambiguous clinical progression (i.e. renewed transfusion dependence and/or stark reduction of general performance).

We show for the first time in AML-patients treated with azacitidine, that further deepening of response after first response, i.e. achievement of BM-blast reduction in terms of $\mathrm{PR}$ or $(\mathrm{m}) \mathrm{CR}$ after $\mathrm{HI}$, seems to translate into significantly longer OS, compared with patients for whom first response was best response (Figures 1 and 2d, Additional file 1: Table S5). Similar results have been shown for MDS-patients treated with azacitidine $[16,35]$. Therefore, in analogy to recommendations for MDS [35,36], these data support the continued use of azacitidine in responding AML-patients.

It is possible, that a larger percentage of AML-MRF and t-AML may be present in our registry-population (Table 1). However, very little recent data is available on the incidence of all WHO-AML-subgroups in adults in Europe [37], and the recently published phase-III trial comparing decitabine vs. conventional care regimen also had a high percentage (35\%) of secondary-AML [30]. In any case, t-AML is known to be associated with dismal prognosis [38], and AML-patients with preceding MDS/ MPN and/or MDS-related cytogenetics (MRC) were shown to have worse OS than AML-NOS [39].

Our registry population includes many elderly and very old patients: $22 \%$ aged $75-79,15 \%$ aged $80-84$ and $8 \% \geq 85$ years, respectively; many of these very old patients would previously have been treated with best supportive care only. Importantly, age had no adverse effect on OS, irrespective of whether a cut-off of $</ \geq 75$ (Additional file 1: Table S4) or $</ \geq 80$ years was chosen (Figure 2a). In addition, only $40 \%$ of patients were untreated prior to azacitidine, whereas $39 \%, 7 \%$ and $6 \%$ were pretreated with conventional chemotherapy, low-dose cytarabine or 'imids', respectively (Table 1). In light of all of the above, we consider our response and OS results particularly relevant.

The absolute number of comorbidities had no adverse effect on OS. Accordingly, the HCT-CI [40], which is based on the number and weighting of comorbidities, could not discriminate patient-groups with different OS (Additional file 1: Table S4), whereas the ECOG-PS [41], which is based on the patient's ability to cope with activities of everyday life, could (Figure 1, Additional file 1: Table S5). In our opinion, high age and presence of comorbidities should not lead to a decision to withhold treatment of azacitidine in favor of BSC, if the patient has an ECOG-PS $<2$.

Azacitidine seems effective in AML, irrespective of BM-blast count (Additional file 1: Table S4, Figure 2b). Lack of correlation between survival and median pretreatment BM-blast count has previously been reported in WHO-AML-patients $(n=40)$ [21]. We believe, that AML-patients with $>30 \%$ BM-blasts should not be precluded from treatment with azacitidine.

Interestingly, patients experiencing hematologic toxicity grade 3-4 in any, one, several, or all cell lineages had significantly better OS (Figure 1). In clinical practice it is important to note that AML-patients may develop a worsening of cytopenia(s) during the first two months prior to azacitidine response, but this does not seem to be associated with an increased rate of infection or hemorrhage $[14,15]$. In fact, even when occurring, these events did not negatively impact OS in our cohort, and neither did non-hematologic toxicity, nor duration of adverse events (Additional file 1: Table S4). The majority of grade 3-4 hematologic adverse events were documented during early treatment cycles, suggesting that (a) patient tolerance to azacitidine increases as treatment continues, (b) a certain amount of aplasia-induction seems necessary before response occurs, and (c) likely reflects the fact that most patients respond after cycle 3. Patients in whom azacitidine dose had to be reduced due to an adverse event even had improved OS in univariate analyses. This effect was however lost in multivariate analysis. It seems fair to state that dose reductions were possible without negative impact on OS. We deduct that the occurrence of adverse events should not lead to permanent treatment discontinuation with azacitidine in most cases. Azacitidine should be continued as planned whenever possible, if necessary with dose reduction or treatment pause.

Achievement of the FDA-approved target-dose of $75 \mathrm{mg} / \mathrm{m}^{2} \times 7$ days did not have a significant effect on OS, and neither did the predominantly applied schedule (Additional file 1: Table S4). Similar results have been shown by others for MDS $[27,40]$. Concerning OS of responding patients only $(\mathrm{n}=70)$, it made no relevant difference which regimen or dose was used in the first cycle, or which regimen or dose was predominantly used over all cycles (Additional file 1: Table S4). The 5-2-2 regimen and/or dose reductions, be it in days (d1-5) and/or dose per day (100 mg flat), seem feasible, safe, and without loss of efficacy. In spite of the impression of 
non-inferiority of alternative schedules and dosages demonstrated by our data, we still recommend initiation of azacitidine in the FDA-approved dosage and regimen.

We acknowledge the limitations of data obtained from a registry and are fully aware, that clinical trials may not be substituted, but only complemented by these. If adequate in size and analyzed carefully, registry data can be of value in supplementing or extending data from trials. The strength of this manuscript lies in the size of the documented patient group, that allows statistical generation of hypothesis, which may be assessed by clinical trials in due time. It was our explicit intention and the design of the registry not to select patients, but in contrast to obtain a widespread view of the use, toxicity and effects of azacitidine in a real-world clinical setting. We believe this is substantiated by the following facts:

a) The population was substantially pretreated;

b) 48 patients (31\%) received only one or two cycles of azacitidine;

c) $90 \%$ of our patient cohort would have been excluded from the pivotal AZA-001 trial due to reasons listed in the discussion of the text;

d) azacitidine was used as last resort, or last line therapy in at least a part of the cohort with a very low life expectancy; these patients would have received best supportive care only before azacitidine was available;

e) $40 \%$ of patients were treated with azacitidine $1^{\text {st }}$ line, as we believed this to be an appropriate option given their disease characteristics, patient profile and in the absence of clinically superior alternatives.

We moderately hypothesize and suggest, that our rather 'aggressive' initiation of treatment, irrespective of comorbidities, bone marrow blast count, age or pretreatment, as well as the 'persistent' continuation of treatment with azacitidine once the drug was commenced (i.e. treatment until overt clinical progression, rather than mere laboratory signs of progression such as stable or slightly rising blast counts in blood or bone marrow) might play a part in our results, although this cannot directly be proven by the data as presented.

\section{Conclusions}

In conclusion, azacitidine seems to be well tolerated and efficacious in WHO-AML-patients in a real-world nontrial clinical setting. The observed median OS of 9.8 months in this largely pretreated cohort including $66 \%$ of patients with bone marrow blasts $>30 \%$ is encouraging. We confirm the previously reported non-essentiallity of CR for prolongation of OS in our cohort of AML-patients treated with azacitidine. In our opinion, age $>80$, number of comorbidities, and/or bone marrow blasts $>30 \%$ should not preclude patients from treatment with this drug, which should be continued as long as response is durable, and onset of overt clinical progression occurs.

\section{Methods}

\section{Registry design and patient eligibility}

WHO-classified AML [42] and treatment with at least one dosage of azacitidine were the sole inclusion criteria (Additional file 2: Figure S2A). No other formal inclusion/exclusion criteria existed. Treatment indication and the decision to offer treatment with azacitidine, as well as dosage, dose reductions/escalations, application route, and administration schedule were exclusively based on the risk/benefit-estimation of the treating physician.

Registry design and timelines can be taken from Additional file 3: Figure S2B. Patients receiving azacitidine prior to EMA-approval, as well as patients with $>30 \% \mathrm{BM}$ blasts were informed of off-label use and gave written informed consent to treatment with azacitidine. Informed consent to allow the collection of personal data was obtained for all retrospectively documented patients who were alive, as well as for all prospectively included patients.

\section{Data collection and monitoring}

Data was entered by physicians and/or trained clinical trial personnel at the respective centers. Central monitoring of all data entered in the eCRF including response evaluations was performed as a quality control measure by LP, in order to assure data integrity and plausibility (cut-off date 21.01.2012). Missing data are low and documented as such. Centers received queries specifying incomplete data or questions to reconfirm data, if necessary.

\section{Assessment of efficacy, safety and endpoints}

Bone marrow punctures/aspirations were performed as clinically necessary; Marrow response, hematologic improvement and overall response were assessed according to current IWG-criteria [19,20]. Central monitoring of response in each cycle was performed. A total of $97 \%$ of our cohort had either AML with $>30 \%$ bone marrow blasts, was refractory to prior conventional chemotherapy or had AML with $20-30 \%$ bone marrow blasts. The remaining $3 \%$ of patients were refractory to other therapies (e.g. low-dose cytarabine). We therefore are certain, that the patients included truly suffered from AML, even without central review of the blast count.

Toxicity and adverse events were assessed according to the NCI Toxicity Criteria (http://ctep.cancer.gov/protocol Development/electronic_applications/ctc.htm) and Common Terminology Criteria for AE (CTCAEv.4) (http://evs. nci.nih.gov/ftp1/CTCAE/About.html). Grade 3-4 cytopenias reported, are those that were documented as adverse events, and thus felt to be a worsening of pre-existing cytopenia by the respective treating physicians. 


\section{Statistical analysis}

Overall survival was assessed using the Kaplan-Meier method. Univariate analyses were performed with logrank tests. Cox-regression stratified on the various factors was used for univariate analyses of risk-factors for OS. For multivariate analysis Cox-regression with stepwise selection (entry-level 0.25; level for keeping the variable 0.15) was used. Univariate analyses were performed and confirmed by two independent statisticians [H.A., B.R.]. The confirmed results were the basis for multivariate analysis. All variables with $P<.05$ in univariate analyses were included in multivariate analysis, except for those cases, where parsimony would have been disrupted due to redundancy in the variables. All analyses were performed with SPSS and SAS. No adjustments were made for multiple testing.

\section{Additional files}

Additional file 1: Table S1. Comparison of overall response rates of al current full publications on AML patients treated with azacitidine. Table S2. Number of AML diagnoses per year in Austria, and patient recruitement to the Austrian Azacitidine Registry (AAR). Table S3. Azacitidine treatment schedule. Table S4. Factors that did not significantly affect overall survival. Table S5. Factors significantly influencing overall survival.

Additional file 2: Figure S1. (CONSORT-Diagram A Describes the design of, and patient eligibility for the Austrian Azacitidine Registry (AAR).

Additional file 3: Figure S1. (CONSORT-Diagram B. Describes the timelines of the Austrian Azacitidine Registry (AAR)

\section{Competing interests}

Consultant or advisory role: Lisa Pleyer, Celgene, Bristol-Myers Squibb, Novartis; Sonja Burgstaller, Celgene; Michael Pfeilstöcker, Celgene, Novartis; Michael Girschikofsky, Mundipharma; Alois Lang, Celgene; Hubert Angermann, Unidata Geodesign $\mathrm{GmbH}$; Reinhard Stauder, Celgene; Alexander Egle, Celgene; Richard Greil, Bristol-Myers-Squibb, Cephalon, Celgene;

Honoraria: Lisa Pleyer, Celgene, Bristol-Myers Squibb, Novartis, AOP Orphan Pharmaceuticals; Thomas Melchardt, Mundipharma; Sonja Burgstaller, Mundipharma, Novartis, AOP Orphan Pharmaceuticals; Michael Pfeilstöcker, Celgene, Novartis; Michael Girschikofsky, Pfizer, Mundipharma; Reinhard Stauder, Ratiopharm, Celgene; Richard Greil, Amgen, Eisai, Mundipharma, Merck, Janssen-Cilag, Genentech, Novartis, Astra-Zeneca, Cephalon, Boehringer-Ingelheim, Pfizer, Roche, Bristol-Myers Squibb, Sanofi Aventis; Peter Krippl, Roche, Amgen, Pfizer, Mundipharma, Galxo Smith Klein, PharmaMar, Astra Zeneca; Alexander Egle, Celgene;

Research funding: Michael Girschikofsky, Pfizer; Reinhard Stauder, Ratiopharm, Novartis, Celgene; Richard Greil, GSK, Amgen, Genentech, Ratiopharm, Celgene, Pfizer, Mundipharma, Cephalon; Peter Krippl, Pfizer, Roche; Alexander Egle, Celgene;

Expert testimony: Peter Krippl, Amgen, Roche, Mundipharma;

Other remuneration: Thomas Melchardt, travel support: Amgen, Sanofi Aventis, Roche, Celgene, BMS, Janssen-Cilag, Böhringer Ingelheim; Reinhard Stauder and Martina Mitrovic were supported by Verein Senioren-Krebshilfe;

\section{Authors' contributions}

All authors had access to all the clinical data, and were kept up-to date with recent results of the registry via oral presentations from $L P$ at regular intervals. All authors participated in regular critical discussions concerning the status and direction of the registry as well as the data to be published. All authors had the opportunity to review the final manuscript prior to submission. The primary and corresponding authors had final responsibility for the decision to submit for publication. Conception and design: $L P, R G$; Statistics and online CRF: HA, LP; Collection and assembly of data: LP, RS, SB, MS, CT, MP, SS, TM, MM, MG, AL, PK, TS, AE, WL, DV, HA, RG; Data analysis and interpretation: $L P, R G ;$ Manuscript writing: LP; Critical revision of the paper: $L P, R G, A E, R S, S B, M S, C T, M P, S S, T M, M M, M G, A L, P K, T S, W L$, DV, HA; Final approval of Manuscript: LP, RS, SB, MS, CT, MP, SS, TM, MM, MG, AL, PK, TS, $A E, W L, D V, H A, R G$; Provision of patients: LP, RS, SB, MS, CT, MP, MG, AL, PK, $T S, A E, W L, D V, R G$

\section{Funding sources}

The AAR is a Registry of the ,Arbeitsgemeinschaft Medikamentöse Tumortherapie' (AGMT) Study Group which served as the responsible sponsor and holds the full and exclusive rights on data. Financial support for the AGMT was received from Celgene. Celgene had no role in study design, data collection, data analysis, data interpretation, or writing of the manuscript.

\section{Non-author contributions}

Barbara Rosettani, a Celgene Employee, contributed solely as an operational/ confirmational statistician, without influencing the design of the analyses or the manuscript. Andrew Brittain, a medical writer at Knowledgepoint360 Group (supported by funding from Celgene), was involved solely for the correct formatting of the figures. He had no influence on planning, writing or interpreting the manuscript.

\section{Author details}

3rd Medical Department with Hematology and Medical Oncology, Hemostaseology, Rheumatology and Infectious Diseases, Laboratory for Immunological and Molecular Cancer Research, Oncologic Center, Paracelsus Medical University Hospital Salzburg, Müllnerhauptstrasse 48, Salzburg 5020, Austria. ${ }^{2}$ Internal Medicine V (Hematology and Oncology), Innsbruck Medical University, Anichstrasse 35, Innsbruck 6020, Austria. ${ }^{3}$ Department for Internal Medicine IV, Hospital Wels-Grieskirchen, Grieskirchnerstrasse 42, 4600, Wels, Austria. ${ }^{4} 1$ st Department of Internal Medicine, Center for Oncology and Hematology, Wilhelminenspital, Montleartstrasse 37, Vienna 1160, Austria. ${ }^{5}$ Department for Hematology and Oncology, LKH Leoben-Eisenerz, Radmeisterstrasse 7-9, Eisenerz 8790, Austria. ${ }^{6}$ IIIrd Medical Department, Hanusch Hospital, Heinrich-Collin-Strasse 30, Vienna 1140, Austria. ${ }^{7} 1$ st Medical Department with Hematology, Stem cell transplantation, Hemostatsis and Medical Oncology, Elisabethinen Hospital, Fadingerstrasse 1, Linz 4010, Austria. ${ }^{8}$ Internal Medicine, Hospital Feldkirch, Carinagasse 47, Feldkirch 6800, Austria. ${ }^{9}$ Department for Internal Medicine, LKH Fuerstenfeld, Krankenhausgasse 1, Fuerstenfeld 8280, Austria. ${ }^{10} 5$ th Medical Department with Oncology und Palliative Medicine, Hietzing, Wolkersbergenstrasse 1, Vienna 1130, Austria. ${ }^{11}$ Department of Hematology, Medical University, Auenbruggerplatz 1, Graz 8036, Austria. ${ }^{12}$ Internal Medicine 3, Center for Hematology and Medical Oncology, General Hospital-Linz GesmbH, Krankenhausstrasse 9, Linz 4021, Austria. ${ }^{13}$ UNIDATA GEODESIGN GmbH, Gaertnergasse 3, Vienna 1030, Austria.

Received: 11 February 2013 Accepted: 22 March 2013 Published: 29 April 2013

\section{References}

1. Lowenberg B: Prognostic factors in acute myeloid leukaemia. Best Pract Res Clin Haematol 2001, 14:65-75.

2. Stone RM: The difficult problem of acute myeloid leukemia in the older adult. CA Cancer J Clin 2002, 52:363-371.

3. Anderson JE, Kopecky KJ, Willman CL, Head D, O'Donnell MR, Luthardt FW, et al: Outcome after induction chemotherapy for older patients with acute myeloid leukemia is not improved with mitoxantrone and etoposide compared to cytarabine and daunorubicin: a Southwest Oncology Group study. Blood 2002, 100:3869-3876.

4. Kantarjian H, O'Brien S, Cortes J, Giles F, Faderl S, Jabbour E, et al: Results of intensive chemotherapy in 998 patients age 65 years or older with acute myeloid leukemia or high-risk myelodysplastic syndrome: predictive prognostic models for outcome. Cancer 2006, 106:1090-1098.

5. Menzin J, Lang K, Earle CC, Kerney D, Mallick R: The outcomes and costs of acute myeloid leukemia among the elderly. Arch Intern Med 2002, 162:1597-1603.

6. Deschler B, de Witte T, Mertelsmann R, Lubbert M: Treatment decision-making for older patients with high-risk myelodysplastic 
syndrome or acute myeloid leukemia: problems and approaches. Haematologica 2006, 91:1513-1522.

7. Kantarjian H, Ravandi F, O'Brien S, Cortes J, Faderl S, Garcia-Manero G, et al: Intensive chemotherapy does not benefit most older patients (age 70 years or older) with acute myeloid leukemia. Blood 2010, 116:4422-4429.

8. Burnett AK, Milligan D, Prentice AG, Goldstone AH, McMullin MF, Hills RK, et al: A comparison of low-dose cytarabine and hydroxyurea with or without all-trans retinoic acid for acute myeloid leukemia and high-risk myelodysplastic syndrome in patients not considered fit for intensive treatment. Cancer 2007, 109:1114-1124.

9. Burnett AK, Russell NH, Culligan D, Cavanagh J, Kell J, Wheatley K, et al: The addition of the farnesyl transferase inhibitor, tipifarnib, to low dose cytarabine does not improve outcome for older patients with AML. Br J Haematol 2012, 158:519-522.

10. Faderl S, Ravandi F, Huang X, Garcia-Manero G, Ferrajoli A, Estrov Z, et al: A randomized study of clofarabine versus clofarabine plus low-dose cytarabine as front-line therapy for patients aged 60 years and older with acute myeloid leukemia and high-risk myelodysplastic syndrome. Blood 2008, 112:1638-1645.

11. Giles F, Rizzieri D, Karp J, Vey N, Ravandi F, Faderl S, et al: Cloretazine (VNP40101M), a novel sulfonylhydrazine alkylating agent, in patients age 60 years or older with previously untreated acute myeloid leukemia. J Clin Oncol 2007, 25:25-31.

12. Harousseau JL, Martinelli G, Jedrzejczak WW, Brandwein JM, Bordessoule D, Masszi T, et al: A randomized phase 3 study of tipifarnib compared with best supportive care, including hydroxyurea, in the treatment of newly diagnosed acute myeloid leukemia in patients 70 years or older. Blood 2009, 114:1166-1173.

13. Tilly H, Castaigne S, Bordessoule D, Casassus P, Le Prise PY, Tertian G, et al: Low-dose cytarabine versus intensive chemotherapy in the treatment of acute nonlymphocytic leukemia in the elderly. J Clin Oncol 1990, 8:272-279.

14. Ziogas DC, Voulgarelis M, Zintzaras E: A network meta-analysis of randomized controlled trials of induction treatments in acute myeloid leukemia in the elderly. Clin Ther 2011, 33:254-279.

15. Silverman LR, McKenzie DR, Peterson BL, Holland JF, Backstrom JT, Beach CL, et al: Further analysis of trials with azacitidine in patients with myelodysplastic syndrome: studies 8421, 8921, and 9221 by the Cancer and Leukemia Group B. J Clin Oncol 2006, 24:3895-3903.

16. Fenaux P, Mufti GJ, Hellstrom-Lindberg E, Santini V, Finelli C, Giagounidis A, et al: Efficacy of azacitidine compared with that of conventional care regimens in the treatment of higher-risk myelodysplastic syndromes: a randomised, open-label, phase III study. Lancet Oncol 2009, 10:223-232.

17. Fenaux P, Mufti GJ, Hellstrom-Lindberg E, Santini V, Gattermann N, Germing $U$, et al: Azacitidine prolongs overall survival compared with conventional care regimens in elderly patients with low bone marrow blast count acute myeloid leukemia. J Clin Oncol 2010, 28:562-569.

18. Grimwade D, Walker H, Oliver F, Wheatley K, Harrison C, Harrison G, et al: The importance of diagnostic cytogenetics on outcome in AML: analysis of 1,612 patients entered into the MRC AML 10 trial. The Medical Research Council Adult and Children's Leukaemia Working Parties. Blood 1998, 92:2322-2333.

19. Cheson BD, Greenberg PL, Bennett JM, Lowenberg B, Wijermans PW, Nimer SD, et al: Clinical application and proposal for modification of the International Working Group (IWG) response criteria in myelodysplasia. Blood 2006, 108:419-425.

20. Cheson BD, Bennett JM, Kopecky KJ, Buchner T, Willman CL, Estey EH, et al: Revised recommendations of the International Working Group for Diagnosis, Standardization of Response Criteria, Treatment Outcomes, and Reporting Standards for Therapeutic Trials in Acute Myeloid Leukemia. J Clin Oncol 2003, 21:4642-4649.

21. Al Ali HK, Jaekel N, Junghanss C, Maschmeyer G, Krahl R, Cross M, et al: Azacitidine in patients with acute myeloid leukemia medically unfit for or resistant to chemotherapy: a multicenter phase $1 / / 1$ study. Leuk Lymphoma 2012, 53:110-117

22. Maurillo L, Venditti A, Spagnoli A, Gaidano G, Ferrero D, Oliva E, et al: Azacitidine for the treatment of patients with acute myeloid leukemia: report of 82 patients enrolled in an Italian compassionate program. Cancer 2012, 118:1014-1022

23. Sudan N, Rossetti JM, Shadduck RK, Latsko J, Lech JA, Kaplan RB, et al: Treatment of acute myelogenous leukemia with outpatient azacitidine. Cancer 2006, 107:1839-1843.
24. Thepot S, Itzykson R, Seegers V, Raffoux E, Quesnel B, Chait Y, et al: Treatment of progression of Philadelphia negative myeloproliferative neoplasms to myelodysplastic syndrome or acute myeloid leukemia by azacitidine: a report on 54 cases on the behalf of the Groupe Francophone des Myelodysplasies. Blood 2010:

25. van der Helm LH, Alhan C, Wijermans PW, van Marwijk KM, Schaafsma R, Biemond BJ, et al: Platelet doubling after the first azacitidine cycle is a promising predictor for response in myelodysplastic syndromes (MDS), chronic myelomonocytic leukaemia (CMML) and acute myeloid leukaemia (AML) patients in the Dutch azacitidine compassionate named patient programme. Br J Haematol 2011, 155:599-606.

26. Gavillet M, Noetzli J, Blum S, Duchosal MA, Spertini O, Lambert JF: Transfusion independence and survival in patients with acute myeloid leukemia treated with 5-azacytidine. Haematologica 2012, 97:1929-1931.

27. Lyons RM, Cosgriff TM, Modi SS, Gersh RH, Hainsworth JD, Cohn AL, et al: Hematologic response to three alternative dosing schedules of azacitidine in patients with myelodysplastic syndromes. J Clin Oncol 2009, 27:1850-1856.

28. Burnett AK, Hills RK, Hunter A, Milligan D, Kell J, Wheatley K, et al: The addition of arsenic trioxide to low-dose Ara-C in older patients with $\mathrm{AML}$ does not improve outcome. Leukemia 2011, 25:1122-1127.

29. Goldstone AH, Burnett AK, Wheatley $K$, Smith AG, Hutchinson RM, Clark RE: Attempts to improve treatment outcomes in acute myeloid leukemia (AML) in older patients: the results of the United Kingdom Medical Research Council AML11 trial. Blood 2001, 98:1302-1311.

30. Quintas-Cardama A, Ravandi F, Liu-Dumlao T, Brandt M, Faderl S, Pierce S, et al: Epigenetic therapy is associated with similar survival compared with intensive chemotherapy in older patients with newly diagnosed acute myeloid leukemia. Blood 2012, 120:4840-4845.

31. Schiller GJ, O'Brien SM, Pigneux A, Deangelo DJ, Vey N, Kell J, et al: Single-agent laromustine, a novel alkylating agent, has significant activity in older patients with previously untreated poor-risk acute myeloid leukemia. J Clin Oncol 2010, 28:815-821.

32. Kantarjian HM, Thomas XG, Dmoszynska A, Wierzbowska A, Mazur G, Mayer J, et al: Multicenter, randomized, open-label, phase III trial of decitabine versus patient choice, with physician advice, of either supportive care or low-dose cytarabine for the treatment of older patients with newly diagnosed acute myeloid leukemia. J Clin Oncol 2012, 30:2670-2677.

33. Silverman LR, Demakos EP, Peterson BL, Kornblith AB, Holland JC, Odchimar-Reissig $R$, et al: Randomized controlled trial of azacitidine in patients with the myelodysplastic syndrome: a study of the cancer and leukemia group B. J Clin Oncol 2002, 20:2429-2440.

34. Pierdomenico F, Almeida A: Efficacy, tolerability and cost benefit of a 5-Day Azacitidine Regimen. Blood 2011, 118:. Ref Type: Generic.

35. Silverman LR, Fenaux P, Mufti GJ, Santini V, Hellstrom-Lindberg E, Gattermann N, et al: Continued azacitidine therapy beyond time of first response improves quality of response in patients with higher-risk myelodysplastic syndromes. Cancer 2011, 117:2697-2702.

36. Ruter B, Wijermans PW, Lubbert M: Superiority of prolonged low-dose azanucleoside administration? Results of 5-aza-2'-deoxycytidine retreatment in high-risk myelodysplasia patients. Cancer 2006, 106:1744-1750.

37. Sant M, Allemani C, Tereanu C, De Angelis R, Capocaccia R, Visser O, et al: Incidence of hematologic malignancies in Europe by morphologic subtype: results of the HAEMACARE project. Blood 2010, 116:3724-3734.

38. Smith SM, Le Beau MM, Huo D, Karrison T, Sobecks RM, Anastasi J, et al: Clinical-cytogenetic associations in 306 patients with therapy-related myelodysplasia and myeloid leukemia: the University of Chicago series. Blood 2003, 102:43-52.

39. Miesner M, Haferlach C, Bacher U, Weiss T, Macijewski K, Kohlmann A, et al: Multilineage dysplasia (MLD) in acute myeloid leukemia (AML) correlates with MDS-related cytogenetic abnormalities and a prior history of MDS or MDS/MPN but has no independent prognostic relevance: a comparison of 408 cases classified as "AML not otherwise specified" (AML-NOS) or "AML with myelodysplasia-related changes" (AML-MRC). Blood 2010, 116:2742-2751.

40. Sorror ML, Maris MB, Storb R, Baron F, Sandmaier BM, Maloney DG, et al: Hematopoietic cell transplantation (HCT)-specific comorbidity index: a new tool for risk assessment before allogeneic HCT. Blood 2005, 106:2912-2919. 
41. Oken MM, Creech RH, Tormey DC, Horton J, Davis TE, McFadden ET, et al: Toxicity and response criteria of the Eastern Cooperative Oncology Group. Am J Clin Oncol 1982, 5:649-655.

42. Swerdlow SH, Harris NL CE (Eds): World Health Organization Classification of Tumours of Haematopoietic and Lymphoid Tissues. Lyon: IARC Press; 2008. Ref Type: Generic.

doi:10.1186/1756-8722-6-32

Cite this article as: Pleyer et al: Azacitidine in patients with WHOdefined AML - Results of 155 patients from the Austrian Azacitidine Registry of the AGMT-Study Group. Journal of Hematology \& Oncology 2013 6:32.

\section{Submit your next manuscript to BioMed Central and take full advantage of:}

- Convenient online submission

- Thorough peer review

- No space constraints or color figure charges

- Immediate publication on acceptance

- Inclusion in PubMed, CAS, Scopus and Google Scholar

- Research which is freely available for redistribution 